\title{
A novel transdermal drug-delivery patch for treating local muscular pain
}

\author{
Chandra S Sharma*,1 \& Mudrika Khandelwal ${ }^{2}$ \\ ${ }^{1}$ Creative \& Advanced Research Based On Nanomaterials (CARBON) Laboratory, Department of Chemical Engineering, Indian \\ Institute of Technology, Hyderabad, Kandi-502285, Telangana, India \\ ${ }^{2}$ Department of Materials Science \& Metallurgical Engineering, Indian Institute of Technology, Hyderabad, Kandi - 502285, \\ Telangana, India \\ *Author for correspondence: cssharma@iith.ac.in
}

First draft submitted: 8 January 2018; Accepted for publication: 4 April 2018; Published online: 3 May 2018

Keywords: diclofenac • electrospun nanofibers $\bullet$ transdermal patch

Superficial nociceptive pains such as myalgia (muscular pain) can have a wide range of causes, from bee stings and burns to trauma, muscle overuse or chronic tensions, and is often associated with inflammation and tissue swelling [1]. Generally, a specific group of drugs called nonsteroidal anti-inflammatory drugs (NSAIDs) can be used for the treatment of local muscular pain and inflammation. The NSAIDs inhibit the activity of cyclooxygenase enzymes (COX-1 and COX-2), thereby controlling the synthesis of thromboxanes and prostaglandins [2]. The cyclooxygenase is an enzyme released during pain and inflammation, whereas thromboxanes and prostaglandins are mediators of vasoconstriction and inflammation, respectively [3]. Some commonly used NSAIDs include aspirin, ibuprofen, naproxen and diclofenac.

Diclofenac is used for the treatment of mild to moderate muscular pain, as well as for alleviating the symptoms of arthritis, menstrual cramps, toothache and migraines/headaches [4]. However, if it is taken orally for a long period of time in high doses, then it may potentially lead to fatal stomach and intestinal bleeding, and perhaps increase the risk of a heart attack or stroke [5]. Today, patients are becoming more and more conscious of such side effects and hence novel and safer ways of pain management are desirable.

Transdermal drug delivery (TDD) can pose as a safer and preferred alternative to oral drug delivery as well as parenteral administration for NSAIDs due to various reasons [6]. One advantage is that the transdermal route avoids the metabolism of the drug inside the liver and its rapid absorption inside the GI tract, thus avoiding the risk of internal bleeding and irritation. Additionally, TDD typically avoids painful administration or other unwanted systemic side effects that may occur with oral administration. TDD systems are designed to deliver a predetermined amount of drug through the stratum corneum and subsequently the systemic circulation [7]. The choice of drug for TDD depends on the size of the drug, as it should efficiently pass through the skin pores. Conventional means of TDD include ointments, creams and gels; however, the major disadvantage of these is the need for frequent application, the inability to determine exact dosage administered and instability. Moreover, strong ointments or their improper usage can potentially lead to skin allergies, dryness, lesions and thinning of the skin [8].

Given this, TDD systems, such as drug-loaded patches, backing films, microneedles, thermal, mechanical and electrical ablation, are often preferred $[9,10]$. Out of these, TDD patches are simple and the most patient-compliant. A few transdermal patches for treating muscular pain are available on the market - most based on either narcotic (Fentanyl) or methyl salicylate (Salonpas), which can cause breathing problems, or toxicity issues [11,12].

\section{Novel diclofenac-loaded TDD patches}

Ideally, TDD systems need to facilitate the permeation of drug through the stratum corneum, systematically deliver the drug and maintain minimal skin contact in order to avoid local irritations, itching and allergies. For this, we have recently developed a cellulose acetate-based nanofibrous transdermal patch that controls and sustains the release of diclofenac sodium salt for a prolonged period of time. These patches minimize direct drug-skin contact, excessive dosing and burst release [13]. The main problems associated with the drug diclofenac are its half-life (1-2 
h), fast absorption (due to hydrophilicity) and its toxicity (due to high loading and frequent dosing). However, these issues are well addressed by these patches due to their capability to effectively control drug release.

The nanofibrous transdermal patches were synthesized by electrospinning techniques. Electrospinning is a fiber generation technique that utilizes a high electric field to produce nanofibers from the polymer solution or melts. Under a high electric field, the polymer gets polarized and the charged polymer threads are drawn toward the oppositely charged collector, resulting in fibers. The nanofibers are collected as a nonwoven fabric, which possesses high surface area and high porosity. We used cellulose acetate solution loaded with diclofenac sodium for electrospinning to obtain nanofibrous patches with uniform encapsulation of the drug. However, this does not alone guarantee a sustained and prolonged release. The surface interaction and wettability of the patch plays a pivotal role in controlling the drug release. One way to control wettability is to pattern the fabric on the surface, which basically alters the surface roughness and thus the contact surface area [14]. We have used nylon meshes of different pore sizes (50,100 and 200 microns) as a template for collecting the nanofibers. A preferential deposition of nanofibers was observed on the nylon threads of the mesh. The fabric thus obtained is a rough replica of the mesh with air pockets at the place of the pores in the mesh. This combination of fibers and gaps offers different contact surface areas and hence different wettability for different meshes [14]. These micropatterned nanofibrous fabrics with different wettability showed different drug release profiles. Hence, we were indeed able to tune wettability to control and sustain drug release for a long period of time. Some of the notable features of these novel TDD patches are listed below.

\section{Sustained (zero order) drug release for up to $12 \mathrm{~h}$}

The release of drug was tested using a membrane that mimics the skin, in a buffer solution with a $\mathrm{pH}$ of 5.5. Here, we were able to achieve zero-order drug release for up to $12 \mathrm{~h}$. During the initial hours (the first $3 \mathrm{~h}$ ) wettability plays a major role in controlling the burst release. After some time, the surface becomes wet and the release is then controlled by porosity. The porosity facilitates the swelling of the nanofibrous mats and diffusion of the drug from the bulk of fiber mat [13].

This transdermal patch may be used to treat local pain however not deep-seated pain. The patch can be effectively used up to $12 \mathrm{~h}$ although we could also observe drug release for up to $48 \mathrm{~h}$.

\section{No burst releases \& frequent dosing}

Only $30 \%$ of the drug was released in the first $3 \mathrm{~h}$, which indicates minimum accumulation of drug at the target site, and thus minimizes if not eliminates any side effects of overdose [13]. As we reduced dosing frequency and also controlled initial burst release, the side effects of the drug due to excessive systemic absorptions should be absent.

\section{Minimal skin contact}

The diclofenac-sodium-loaded cellulose acetate nanofibers were found to be slender, elongated and uniform without any beads. The drug molecules were uniformly encapsulated into the cellulose acetate biopolymer. This ensures minimum direct skin contact with the drug, reducing the chances of skin irritations, itching and allergies. In addition, typically ointments and gels need to be applied to larger areas of skin, which can lead to larger skin reactions; however, these patches need only a small area to be in contact and thus minimizes any unwanted interactions.

\section{Tunable wettability}

For this delivery method, there is a facile and single-step technique to tune wettability of the cellulose acetate fabric. Cellulose acetate is a water-insoluble, hydrophilic and biocompatible polymer. We have shown that the surface contact angle of cellulose acetate nanofibers can be varied from $30^{\circ}$ (for unpatterned) to over $135^{\circ}$ by nylon mesh template assisted electrospinning. The hydrophobicity is induced because of the nonwetting capillary pressure developed due to the air trapped inside the air gaps of the patterned mat [14]. This nonwetting pressure decreases with the increase in the spacing of pattern, that is toward higher mesh size.

\section{Commercialization}

The packaging of the patch plays a crucial role in bringing the product to the market. The packaging comprises of a liner, drug-loaded mat, an adhesive and a backing [15]. A liner protects the patch during storage and should be removed before using the patch. An adhesive holds the components of the drug-loaded mat together and may 
carry drug in itself. An adhesive should hold the patch for the desired time and should come out easily after use without causing pain. Backing protects the patch from the outer environment [16]. The selection of each packaging component shall be based on the desired properties as well as the human and environmental friendliness. We are in a process of preparing a prototype that can be first tested on animals. It is interesting and promising to see that currently only a few patches for non-narcotic drugs or those with NSAIDs are available for pain management. FLECTOR is one such available TDD patch. However, it also comes with a warning to minimize usage duration [17], maybe due to high drug loading. Nanofibrous patches may therefore be a promising alternative.

\section{Future perspective}

Nanofiber-based TDD systems may lead to more painless, efficient, nontoxic and patient-compliant drug delivery. Various other drugs to diclofenac may also be incorporated for targeting a variety of skin disorders. For instance, anticancer drugs may be encapsulated to treat basal cell cancer or an antimicrobial drug to target cellulitis, boils, abscesses, etc. Overall, this micropatterned nanofiber-based transdermal patch has enormous potential to be used not only for effective and focused pain management but also for other conditions requiring transdermal drug release. These patches therefore have potential as a future sophisticated TDD strategy.

\section{Financial \& competing interest disclosure}

The authors have no relevant affiliations or financial involvement with any organization or entity with a financial interest in or financial conflict with the subject matter or materials discussed in the manuscript. This includes employment, consultancies, honoraria, stock ownership or options, expert testimony, grants or patents received or pending, or royalties.

No writing assistance was utilized in the production of this manuscript.

\section{References}

1. Søgaard K, Sjøgaard G. Physical activity as cause and cure of muscular pain: evidence of underlying mechanisms. Exerc. Sport Sci. Rev. 45(3), 136-145 (2017).

2. Díaz-González F, Sánchez-Madrid F. NSAIDs: learning new tricks from old drugs. Eur. J. Immunol. 45(3), 679-686 (2015).

3. Newton RF, Roberts SM. History, nomenclature and potential uses of prostaglandins and thromboxanes in the clinic. In: Prostaglandins and Thromboxanes: Butterworths Monographs in Chemistry. Newton RF, Roberts SM (Ed.). Butterworth Scientific, London, UK, 1-7, (1982).

4. Zhang Y, Cun D, Kong X, Fang L. Design and evaluation of a novel transdermal patch containing diclofenac and teriflunomide for rheumatoid arthritis therapy. Asian J. Pharm. Sci. 9, 251-259 (2014).

5. Harirforoosh S, Asghar W, Jamali F. Adverse effects of nonsteroidal antiinflammatory drugs: an update of gastrointestinal, cardiovascular and renal complications. J. Pharm. Pharm. Sci. 16(5), 821-847 (2014).

6. Paudel KS, Milewski M, Swadley CL, Brogden NK, Ghosh P, Stinchcomb AL. Challenges and opportunities in dermal/transdermal delivery. Ther. Deliv. 1(1), 109-131 (2010).

7. Akhtar NI. Microneedles: an innovative approach to transdermal delivery - a review. Int. J. Pharm. Pharm. Sci. 6, 18-25 (2014).

8. Brown MB, Martin GP, Jones SA, Akomeah FK. Dermal and transdermal drug delivery systems: current and future prospects. Drug Deliv. 13(3), 175-187 (2006).

9. Prausnitz MR, Langer R. Transdermal drug delivery. Nat. Biotechnol. 26(11), 1261-1268 (2008).

10. Alkilani AZ, McCrudden MT, Donnelly RF. Transdermal drug delivery: innovative pharmaceutical developments based on disruption of the barrier properties of the stratum corneum. Pharmaceutics 7(4), 438-470 (2015).

11. Bakovic M, Nestic M, Mayer D. Death by band-aid: fatal misuse of transdermal fentanyl patch. Int. J. Legal Med. 129(6), 1247-1252 (2015).

12. The 5 best pain relieving patches.www.top5reviewed.com/pain-relieving-patches/

13. Adepu S, Gaydhane MK, Kakunuri M, Sharma CS, Khandelwal M, Eichhorn SJ. Effect of micropatterning induced surface hydrophobicity on drug release from electrospun cellulose acetate nanofibers. Appl. Surf. Sci. 426, 755-762 (2017).

14. Kakunuri M, Wanasekara ND, Sharma CS, Khandelwal M, Eichhorn SJ. Three-dimensional electrospun micropatterned cellulose acetate nanofiber surfaces with tunable wettability. J. Appl. Polym. Sci. 134, 44709-44715 (2017).

15. FABRICO. Medical converting and packaging for transdermal delivery of cosmeceuticals, nutraceuticals, and aromatherapy.www.fabrico.com/sites/default/files/Import_10/Import/FAB_Application_Sheet_Medical_4_Cosmeceuticals.pdf

16. Lv S, Quan P, Liu X, Fang L. Effect of backing films on the transdermal delivery of cyclobenzaprine patch. Asian J. Pharm. Sci. 11, 780-783 (2016).

17. Flector patch. Flector patch Diclofenac Epolamine Topical Patch 1.3\%.www.flectorpatch.com 
